Case Report

\title{
Tofacitinib for the Treatment of Severe Interstitial Lung Disease Related to Rheumatoid Arthritis
}

\author{
Caterina Vacchi, ${ }^{1}$ Andreina Manfredi, ${ }^{2}$ Giulia Cassone, ${ }^{1}$ Stefania Cerri, ${ }^{3}$ \\ Giovanni Della Casa, ${ }^{4}$ Dario Andrisani, ${ }^{1}$ Carlo Salvarani, ${ }^{2}$ and Marco Sebastiani ${ }^{2}{ }^{2}$ \\ ${ }^{1} \mathrm{PhD}$ Program in Clinical and Experimental Medicine, University of Modena and Reggio Emilia, Via Del Pozzo 71, \\ Modena 41125, Italy \\ ${ }^{2}$ Rheumatology Unit, University of Modena and Reggio Emilia, Via Del Pozzo 71, Modena 41125, Italy \\ ${ }^{3}$ Respiratory Diseases Unit and Centre for Rare Lung Diseases, University of Modena Reggio Emilia, Via Del Pozzo 71, \\ Modena 41125, Italy \\ ${ }^{4}$ Radiology Unit, University of Modena and Reggio Emilia, Via Del Pozzo 71, Modena 41125, Italy
}

Correspondence should be addressed to Marco Sebastiani; marco.sebastiani@unimore.it

Received 9 December 2020; Accepted 14 April 2021; Published 22 April 2021

Academic Editor: Massimo Conese

Copyright ( $) 2021$ Caterina Vacchi et al. This is an open access article distributed under the Creative Commons Attribution License, which permits unrestricted use, distribution, and reproduction in any medium, provided the original work is properly cited.

\begin{abstract}
Rheumatoid arthritis (RA) is a chronic systemic inflammatory disease characterized by chronic symmetrical erosive synovitis and extra-articular manifestations, including interstitial lung disease (ILD), whose treatment is nowadays challenging due to high infectious risk and possible pulmonary iatrogenic toxicity. Janus kinase inhibitors, namely, tofacitinib, baricitinib, and upadacitinib, are the latest drug class for the treatment of RA with a good safety profile. We present the case of a patient with RA-ILD successfully treated with tofacitinib. A 52-year-old man was referred to our multidisciplinary clinic for rheumatic and pulmonary diseases for an active erosive seropositive RA and progressive ILD. Previous treatments were GC, hydroxychloroquine, methotrexate, etanercept, withdrawn after ILD detection, and tocilizumab, discontinued due to relapsing infections. After our evaluation, we proposed rituximab in addition to low-dose GC and hydroxychloroquine, ineffective on joint involvement. Therefore, we proposed tofacitinib which allowed us to control joint involvement, stabilize ILD improving respiratory symptoms, and manage the frequent infectious episodes that occurred initially. The short half-life and rapid-acting of tofacitinib are two helpful characteristics regarding this aspect. Despite limited data from randomized trials and real-life, tofacitinib could represent a safe therapeutic option for RA-ILD patients. Longitudinal studies are required to confirm this encouraging report.
\end{abstract}

\section{Introduction}

Rheumatoid arthritis (RA) is a chronic systemic inflammatory disease affecting $0.5-1 \%$ of the population worldwide. It is characterized by chronic symmetrical erosive synovitis and sometimes by extra-articular manifestations [1], including interstitial lung disease (ILD), which represents the most common lung involvement [2]. ILD significantly affects therapeutic approach, quality of life, morbidity, and mortality of RA patients, with an estimated prevalence ranging from 4 to $30 \%[2,3]$.
Among the main risk factors for the development of ARILD, we consider high titles of rheumatoid factor (RF) and anticitrullinated protein antibodies (ACPA), male sex, and cigarette smoking history [4]. Sometimes lung interstitial involvement is early and represents the only sign of disease. In these cases, a clinical and serological follow-up may lead to evidence of autoimmune disease later in time [5].

In AR patients with lung involvement, the radiological UIP pattern is not only the most frequent one (about $60-70 \%)$ but also the most associated with poor prognosis $[1,6]$. 
The treatment of patients with RA and concomitant ILD is nowadays challenging and mainly based on glucocorticoids (GC) and immunosuppressants [7]. Therapeutic options in these patients are complicated by the possible pulmonary toxicity of many disease modifying antirheumatic drugs (DMARDs) and by their unclear efficacy on pulmonary involvement [7]. The scientific community is constantly looking for therapeutic alternatives to control RA activity avoiding lung toxicity and, possibly, influencing the natural history of RA-ILD. Furthermore, RA-ILD treatment should be safe and easy to manage in case of infectious adverse events, giving the higher infectious risk in ILD patients [8].

Small-molecule Janus kinase inhibitors (JAK-i), namely, tofacitinib, baricitinib, and upadacitinib, are the latest drug class of DMARDs commercialized for the treatment of RA with a good safety profile [9-13].

Here, we present the case of a patient affected with progressive RA-ILD successfully treated with tofacitinib.

\section{Case Report}

A 52-year-old man, former smoker (45 pack years), was referred to our multidisciplinary outpatient clinic for rheumatic and rare lung diseases for an active RA associated with progressive ILD. His past clinical history revealed systemic arterial hypertension, dyslipidaemia, nonalcoholic fatty liver disease, and class II obesity (BMI: 37).

Diagnosis of RA was performed in April 2012. RF and ACPA were positive (42 and $300 \mathrm{U} / \mathrm{mL}$, respectively), while antinuclear antibodies (ANA), extractable nuclear antigens (ENA), and antineutrophil cytoplasmic antibodies (ANCA) were negative.

Arthritis was aggressive and rapidly erosive at hands and wrists. The patient had been initially treated with methotrexate (MTX) $15 \mathrm{mg}$ weekly, effective but replaced with hydroxychloroquine (HCQ) $400 \mathrm{mg}$ daily for the desire of paternity in December 2012. Since HCQ became ineffective, etanercept (ETA) $50 \mathrm{mg}$ weekly was added in April 2014.

Fourteen months later, ILD was casually detected (Figure 1), and ETA was replaced with subcutaneous tocilizumab (TCZ) $162 \mathrm{mg}$ weekly. This latter drug was stopped after few months for relapsing infections, mainly involving the urinary and lower respiratory tract (Figure 1).

In October 2017, due to a deterioration of respiratory symptoms, he was referred for the first time to our multidisciplinary outpatient clinic, which includes a rheumatologist and a pulmonologist. At that time, he presented severe restrictive ventilatory impairment, with a forced vital capacity (FVC) of $52 \%$ of the predicted value and a severe reduction in gas exchange with a diffusion capacity for carbon monoxide (DLCO) of 33\% of the predicted value. Moreover, he desaturated at 6-minute walking test (nadir reached after two minutes with an oxygen saturation of $87 \%)$. As far as RA activity, he showed arthritis of wrists and II-III right metacarpophalangeal; C-reactive protein (CPR; $50 \mathrm{mg} / \mathrm{L}$ ) and erythrocyte sedimentation rate (ESR; $50 \mathrm{~mm} /$ h) were both increased, with a 28 -joint disease activity score (DAS-28 CRP) of 5.27.
At chest high-resolution computed tomography (HRCT), a pattern of usual interstitial pneumonia was described, characterized by reticular abnormalities and honeycombing aspects, particularly at the right lower lobe (Figure 2).

At that time, he was taking HCQ and low-dose prednisone ( $5 \mathrm{mg}$ daily).

According to the clinical picture, we prescribed oxygen supplementation and proposed treatment with rituximab (1000 mg every other week for 2 intravenous infusions), performed in December 2017, maintaining treatment with prednisone and HCQ. Two months later, arthritis was still active, involving bilateral MCF and proximal interphalangeal joints of hands, wrists, and ankles associated with ESR and CRP elevation $(45 \mathrm{~mm}$ and $60 \mathrm{mg} / \mathrm{L}$, respectively).

After multidisciplinary discussion with a pulmonologist, in March 2018, we prescribed tofacitinib $5 \mathrm{mg}$ twice daily. Vaccination for varicella zoster virus (VZV) was not performed because of the immunosuppressive condition of the patient.

One month later, the patient presented an infection of the upper respiratory tract, successfully treated with antibiotic therapy; tofacitinib was discontinued for ten days. Despite other few respiratory and urinary infectious episodes, RA remission was obtained after 3 months. Disease flares appeared when tofacitinib was discontinued, but every time, a rapid arthritis control was obtained with the reintroduction of tofacitinib.

From August 2018 to March 2020, we observed a stable RA remission, and no other infections were reported by the patient.

Despite an initial radiological progression detected at HRCT in November 2018, respiratory symptoms improved and lung function (FVC and DLCO) remained stable over the time (Figure 1).

\section{Discussion}

ILD is a serious pulmonary complication of RA characterized by a significant impact on morbidity and mortality, representing a current therapeutic challenge because of the possible pulmonary toxicity of many traditional and biological DMARDs, their unclear efficacy on pulmonary disease, and the higher infectious risk in comparison to nonILD RA patients $[7,8]$.

JAK-is have recently emerged in clinical practice for the treatment of RA, and they are recommended by the European League against Rheumatism (EULAR) in patients failing an initial treatment with MTX or other conventional DMARDs with poor prognostic factors [14].

Despite limited data, no relevant safety concerns in relation to both ILD onset and progression are emerged for tofacitinib $[9,11,15,16]$.

A post hoc analysis on 7061 RA patients receiving tofacitinib from clinical trials and long-term extension studies reported an incidence rate for ILD with both tofacitinib doses of 0.18 per 100 patient-years [17]. 


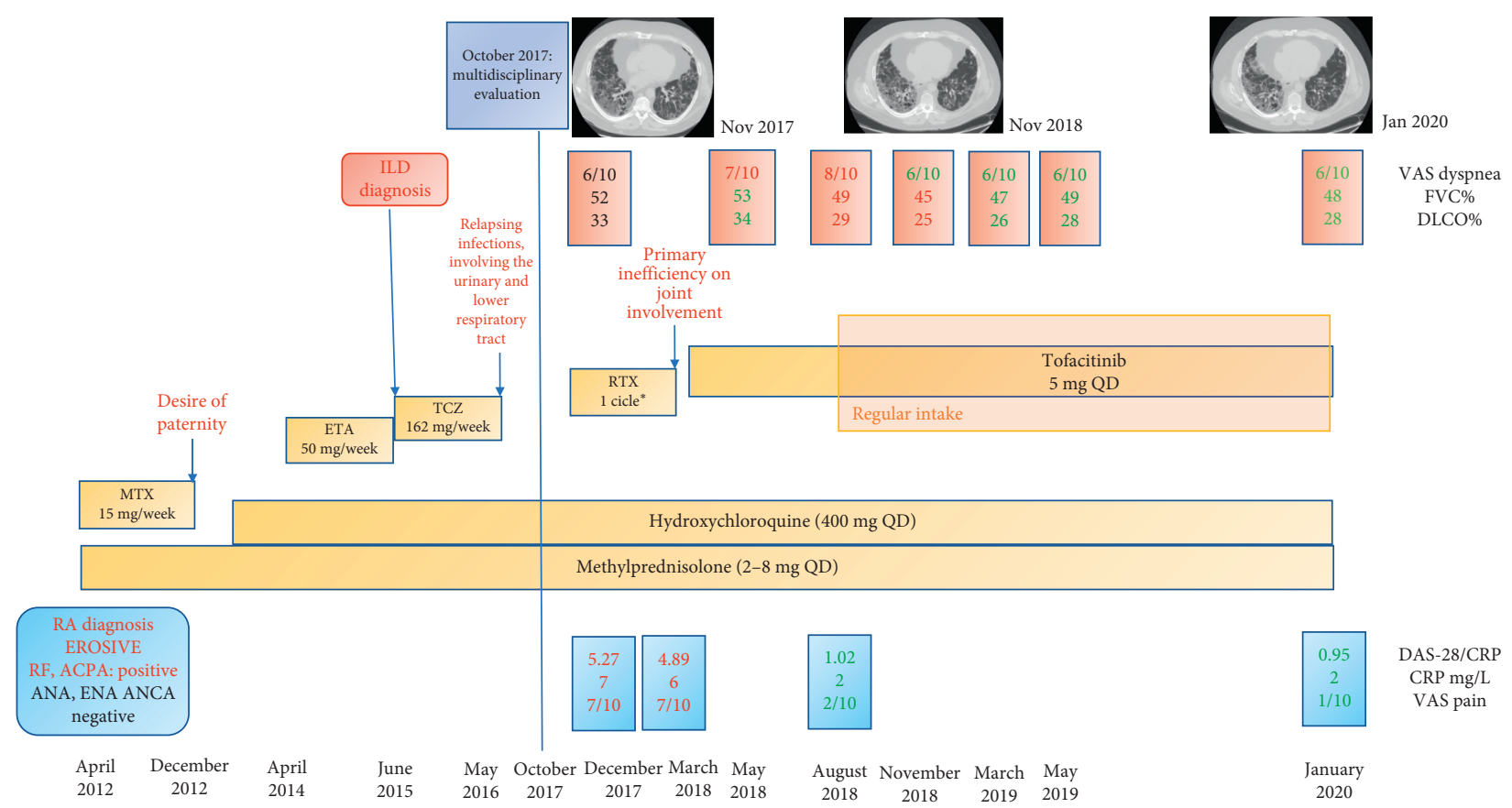

FIGURE 1: Clinical course and therapeutic strategy. ILD: interstitial lung disease; MTX: methotrexate; ETA: etanercept; TCZ: tocilizumab; RTX: rituximab; VAS: Visual Analogue Scale; FVC: forced vital capacity; DLCO: diffusing capacity of the lung for carbon monoxide; RA: rheumatoid arthritis; CRP: C-reactive protein; RF: rheumatoid factor; ACPA: anticitrullinated protein antibodies; ANA: antinuclear antibodies; ENA: extractable nuclear antigen; ANCA: antineutrophil cytoplasmic antibodies; DAS-28: disease activity score on 28 joints.

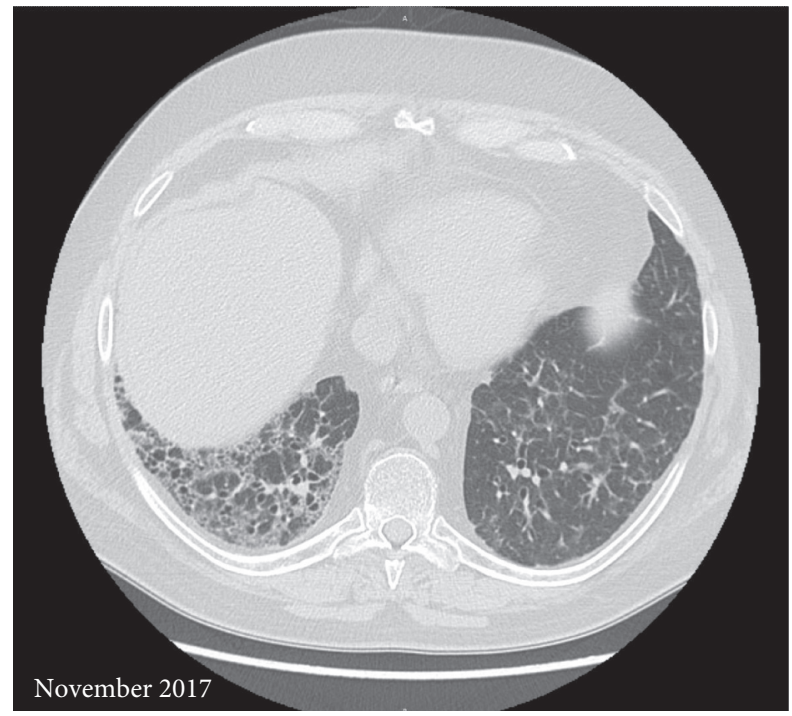

(a)

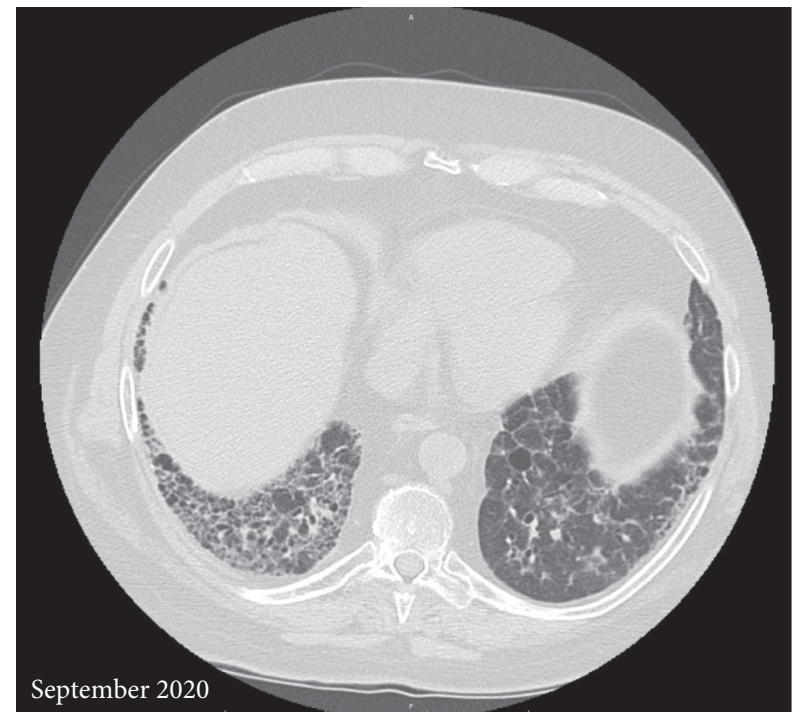

(b)

Figure 2: Chest high-resolution computed tomography: November 2017 compared to last follow-up, September 2020. Reticular abnormalities, honeycombing aspects, and traction bronchiectasis compatible with usual interstitial pneumonia. Despite an initial progression, interstitial lung disease showed a substantial stability at the last follow-up.

Few real-life data are up to now available. Among 15 RAILD patients treated with tofacitinib, none showed worsening of dyspnoea, while improvement was reported in some cases; moreover, respiratory function and DLCO remained stable in the majority of subjects evaluated, while 4 patients improved [18].
In another study, 3 RA-ILD patients were successfully treated with tofacitinib without pulmonary adverse events in an 8-12-month period [19].

About the possible effect on ILD, some case reports have recently been published in which tofacitinib appears to be effective on ILD related to antimelanoma differentiation- 
associated 5 gene antibody-positive dermatomyositis and antisynthetase syndrome [20-23].

Tofacitinib has been demonstrated to be able to reduce the progression of zymosan-induced ILD in SKG mice, a murine model of RA. Tofacitinib significantly suppressed the progression of ILD compared to control by expanding myeloid-derived suppressor cells and suppressing Th17 cells' proliferation and differentiation, suggesting a potential therapeutic effect of tofacitinib for RA-ILD [24].

However, other in vitro studies reported that the inhibition of JAK2, but not the selective JAK1/JAK3 pathway, significantly reduced IL-17A-induced fibrogenic response in fibroblast from IPF and RA-ILD patients [25].

Rituximab was proposed as a therapeutic option for RAILD, even if it has been associated with an increased risk of infections in patients with RA-ILD.

Unfortunately, in our patient, rituximab was ineffective on arthritis and ILD, and it could have contributed to the infectious adverse events showed by the patient in the next six months [26].

The short half-life and rapid-acting of tofacitinib are two characteristics that we have considered for the treatment of our patient. Moreover, we took advantage by an intermittent therapy when the infectious events appeared obtaining a rapid clearance of the drug. On the other side, the patient also showed a rapid flare of disease after tofacitinib discontinuation, requiring a temporary increase of prednisone dose.

\section{Conclusion}

Despite limited data from clinical trials and real-life, tofacitinib could represent a therapeutic option for RA-ILD patients, with a good safety profile. Longitudinal studies are required to confirm this encouraging report.

\section{Conflicts of Interest}

The authors declare no conflicts of interest.

\section{Authors' Contributions}

Sebastiani Marco conceptualized the study, was responsible for methodology, reviewed and edited the manuscript, and was in charge of the case; Vacchi Caterina wrote the original draft, was responsible for methodology, and was in charge of the case; Cassone Giulia, Manfredi Andreina, Andrisani Dario, Della Casa Giovanni, Cerri Stefania, and Salvarani Carlo were in charge of the case and wrote the original draft.

\section{References}

[1] D. N. O’Dwyer, M. E. Armstrong, G. Cooke, J. D. Dodd, D. J. Veale, and S. C. Donnelly, "Rheumatoid arthritis (RA) associated interstitial lung disease (ILD)," European Journal of Internal Medicine, vol. 24, pp. 597-603, 2013.

[2] T. Bongartz, C. Nannini, Y. F. Medina-Velasquez et al., "Incidence and mortality of interstitial lung disease in rheumatoid arthritis: a population-based study," Arthritis \& Rheumatism, vol. 62, no. 6, pp. 1583-1591, 2010.
[3] A. Manfredi, G. Cassone, S. Cerri et al., "Diagnostic accuracy of a velcro sound detector (vector) for interstitial lung disease in rheumatoid arthritis patients: the inspirate validation study (interstitial pneumonia in rheumatoid arthritis with an electronic device)," BMC Pulmonary Medicine, vol. 19, no. 1, p. 111, 2019.

[4] D. van der Woude and A. H. M. van der Helm-van Mil, "Update on the epidemiology, risk factors, and disease outcomes of rheumatoid arthritis," Best Practice \& Research Clinical Rheumatology, vol. 32, no. 2, pp. 174-187, 2018.

[5] T. Bahmer, M. Romagnoli, F. Girelli, M. Claussen, and K. F. Rabe, "The use of auto-antibody testing in the evaluation of interstitial lung disease (ILD) - a practical approach for the pulmonologist," Respiratory Medicine, vol. 113, pp. 80-92, 2016.

[6] P. P. T. e. S. Torres, M. F. Rabahi, M. A. C. Moreira, G. d. S. P. Meirelles, and E. Marchiori, "Usual interstitial pneumonia: typical, possible, and "inconsistent" patterns," Jornal Brasileiro de Pneumologia, vol. 43, no. 5, pp. 393-398, 2017.

[7] G. Cassone, A. Manfredi, C. Vacchi et al., "Treatment of rheumatoid arthritis-associated interstitial lung disease: lights and shadows," Journal of Clinical Medicine, vol. 9, no. 4, p. 1082, 2020.

[8] M. Sebastiani, A. Manfredi, G. Cassone, G. Sandri, S. Cerri, and C. Ferri, "Interstitial lung disease is associated to infections of lower respiratory tract in immunocompromised rheumatoid arthritis patients," Clinical and Experimental Rheumatology, vol. 35, p. 542, 2017.

[9] M. Harigai, "Growing evidence of the safety of JAK inhibitors in patients with rheumatoid arthritis," Rheumatology, vol. 58, no. 1, pp. i34-i42, 2019.

[10] R. F. van Vollenhoven, R. Fleischmann, S. Cohen et al., "Tofacitinib or adalimumab versus placebo in rheumatoid arthritis," New England Journal of Medicine, vol. 367, no. 6, pp. 508-519, 2012.

[11] R. Fleischmann, J. Kremer, J. Cush et al., "Placebo-controlled trial of tofacitinib monotherapy in rheumatoid arthritis," New England Journal of Medicine, vol. 367, no. 6, pp. 495-507, 2012.

[12] P. C. Taylor, E. C. Keystone, D. van der Heijde et al., "Baricitinib versus placebo or adalimumab in rheumatoid arthritis," New England Journal of Medicine, vol. 376, no. 7, pp. 652-662, 2017.

[13] A. Rubbert-Roth, J. Enejosa, A. L. Pangan et al., "Trial of upadacitinib or abatacept in rheumatoid arthritis," New England Journal of Medicine, vol. 383, no. 16, pp. 1511-1521, 2020.

[14] J. S. Smolen, R. B. M. Landewé, J. W. J. Bijlsma et al., "EULAR recommendations for the management of rheumatoid arthritis with synthetic and biological disease-modifying antirheumatic drugs: 2019 update," Annals of the Rheumatic Diseases, vol. 79, 2020.

[15] E. Lilly, Elli_Lilly_and_Company. Japanese package inserts of baricitinib, 2020, http://www.info.pmda.go.jp/go/pack/ 3999043F1020_1_02.

[16] http://www.info.pmda.go.jp/go/pack/3999034F1020_1_09/, P. Pfizer, Japanese package inserts of tofacitinib, 2020.

[17] G. Citera, E. Mysler, H. Madariaga et al., "Incidence rates of interstitial lung disease events in tofacitinib-treated rheumatoid arthritis patients," JCR: Journal of Clinical Rheumatology, 2020.

[18] M. Bejarano, M. N. Tamborenea, M. A. Goñi et al., “Ab0418 interstitial lung disease in patients with rheumatoid arthritis treated with tofacitinib," Abstracts Accepted for Publication, vol. 78, p. 1672, 2019. 
[19] L. M. Saldarriaga-Rivera and V. J. López-Villegas, "Inhibidor de Janus quinasas como opción terapéutica en artritis reumatoide y enfermedad pulmonar intersticial asociada: reporte de 4 casos," Revista Colombiana de Reumatología, vol. 26, no. 2, pp. 137-139, 2019.

[20] S.-i. Ohmura, T. Yamabe, and T. Naniwa, "Successful dose escalation of tofacitinib for refractory dermatomyositis and interstitial lung disease with anti-melanoma differentiationassociated gene 5 antibodies," Modern Rheumatology Case Reports, vol. 5, no. 1, pp. 76-81, 2020.

[21] Y. Ishikawa, T. Kasuya, M. Fujiwara, and Y. Kita, "Tofacitinib for recurrence of antimelanoma differentiation-associated gene 5 antibody-positive clinically amyopathic dermatomyositis after remission," Medicine, vol. 99, no. 37, Article ID e21943, 2020.

[22] S. Wendel, N. Venhoff, B. C. Frye et al., "Successful treatment of extensive calcifications and acute pulmonary involvement in dermatomyositis with the Janus-Kinase inhibitor tofacitinib - a report of two cases," Journal of Autoimmunity, vol. 100, pp. 131-136, 2019.

[23] M. Pineton de Chambrun, B. Hervier, S. Chauveau, Y. Tandjaoui-Lambiotte, A. Combes, and Y. Uzunhan, "Tofacitinib in antisynthetase syndrome-related rapidly progressive interstitial lung disease," Rheumatology, vol. 59, no. 12, pp. e142-e143, 2020.

[24] S. Sendo, J. Saegusa, H. Yamada, K. Nishimura, and A. Morinobu, "Tofacitinib facilitates the expansion of myeloid-derived suppressor cells and ameliorates interstitial lung disease in SKG mice," Arthritis Research \& Therapy, vol. 21, no. 1, p. 184, 2019.

[25] J. Zhang, D. Wang, L. Wang et al., "Profibrotic effect of IL-17A and elevated IL-17RA in idiopathic pulmonary fibrosis and rheumatoid arthritis-associated lung disease support a direct role for IL-17A/IL-17RA in human fibrotic interstitial lung disease," American Journal of Physiology-Lung Cellular and Molecular Physiology, vol. 316, no. 3, pp. L487-L497, 2019.

[26] M. Y. Md Yusof, A. Kabia, M. Darby et al., "Effect of rituximab on the progression of rheumatoid arthritis-related interstitial lung disease: 10 years' experience at a single centre," Rheumatology, vol. 56, no. 8, pp. 1348-1357, 2017. 